\title{
Utilization of Acupuncture Services in the Emergency Department Setting: A Quality Improvement Study
}

John R. Burns

Jessica J. F. Kram

Vashir Xiong

Jeanne M. Stark Casadont

Tiffany A. Mullen

Nancy Conway

Dennis J. Baumgardner

Follow this and additional works at: https://aah.org/jpcrr

Part of the Alternative and Complementary Medicine Commons, Community Health and Preventive Medicine Commons, Emergency Medicine Commons, Health and Medical Administration Commons, Integrative Medicine Commons, and the Patient Safety Commons

\section{Recommended Citation}

Burns JR, Kram JJ, Xiong V, Stark Casadont JM, Mullen TA, Conway N, Baumgardner DJ. Utilization of acupuncture services in the emergency department setting: a quality improvement study. J Patient Cent Res Rev. 2019;6:172-8. 10.17294/2330-0698.1688

Published quarterly by Midwest-based health system Advocate Aurora Health and indexed in PubMed Central, the Journal of Patient-Centered Research and Reviews (JPCRR) is an open access, peer-reviewed medical journal focused on disseminating scholarly works devoted to improving patient-centered care practices, health outcomes, and the patient experience. 


\title{
Utilization of Acupuncture Services in the Emergency Department Setting: A Quality Improvement Study
}

\author{
John R. Burns, DPT, MSOM, ${ }^{1}$ Jessica J. F. Kram, MPH, ${ }^{2,3}$ Vashir Xiong, MSOM, LAc, ${ }^{1}$ Jeanne M. \\ Stark Casadont, BSN, RN, MSOM, LAc, ${ }^{1}$ Tiffany A. Mullen, DO,${ }^{1}$ Nancy Conway, MS, ${ }^{1}$ Dennis J. \\ Baumgardner, $\mathrm{MD}^{2,3,4}$ \\ ${ }^{1}$ Department of Integrative Medicine, Aurora Sinai Medical Center, Aurora Health Care, Milwaukee, Wl; ${ }^{2}$ Center for \\ Urban Population Health, Milwaukee, WI; ${ }^{3}$ Department of Family Medicine, Aurora UW Medical Group, Aurora Health \\ Care, Milwaukee, WI; ${ }^{4}$ Department of Family Medicine and Community Health, University of Wisconsin School of \\ Medicine and Public Health, Madison, WI
}
Purpose Patients often present to the emergency department (ED) for pain. As opioid fatalities rise, alternative treatments are warranted for pain management. Acupuncture, a nonpharmacological treatment involving the insertion of needles into skin or tissue at specific points within the body, may help to decrease acute pain. Our study aimed to assess the utilization and impact of acupuncture in the ED for acute pain management.

Methods We conducted a retrospective analysis of purposefully collected quality improvement data. Patients who were $\geq 18$ years old and who presented to the ED at an urban medical center in Wisconsin during 2017 were offered acupuncture services based on their emergency severity index (ESI; range: highest severity [1] - lowest severity [5]), reason for visit, and physician approval. Paired t-tests were used to examine mean differences between pre- and post-acupuncture pain, stress, anxiety, and nausea scores (range: none [0] - worst [10]). Multivariable regression models also were constructed.

Results A total of 379 patients received acupuncture. Patients presented predominately with an ESI score of 3 $(68.9 \%)$ or $4(24.8 \%) ; 46.4 \%$ received opioids in the ED. Mean pre- and post-acupuncture pain scores significantly differed $(6.5$ vs $3.4 ; P<0.001)$; receiving opioids during the $E D$ visit was not associated with improved pain scores $(P=0.948)$. Stress ( 5.7 vs 1.9$)$, anxiety (4.8 vs 1.6$)$, and nausea (1.6 vs 0.6$)$ scores also improved $(P<0.001)$ following acupuncture.

Conclusions Emergency department acupuncture significantly decreased pain, stress, anxiety, and nausea. Our findings support a larger randomized controlled trial to further assesses the impact of acupuncture for acute pain in other ED settings. (J Patient Cent Res Rev. 2019;6:172-178.)

Keywords acupuncture; acute pain; emergency department; nonpharmacological treatment; quality improvement

7 he United States is currently in the midst of a pain management crisis and an opioid prescription catastrophe due to increases in substance abuse and opioid-related deaths, according

Correspondence: John R. Burns, DPT, MSOM, Aurora Sinai Medical Center, 1020 N. 12th Street, 4th Floor, Milwaukee, WI 53233 (john.burns@aurora.org) to a white paper by the Academic Consortium for Integrative Medicine and Health's Pain Task Force. ${ }^{1}$ In 2017, the U.S. Department of Health and Human Services declared the opioid epidemic a "public health emergency," as an estimated 116 opioid-related drug overdoses occurred every day, with 11.5 million people also misusing prescription opioids in $2016 .^{2}$

To address the pain management crisis and the opioid prescription epidemic, the Department of Defense, 
Veterans Health Administration, National Institutes of Health, Food and Drug Administration, and Centers for Disease Control and Prevention are taking action to recommend a shift away from the reliance on opioids for managing pain. ${ }^{1}$ This undertaking, aimed at transforming pain care in the United States to include effective nonpharmacological strategies, may reduce the risks of the aforementioned adverse events. ${ }^{1}$

In the face of rising adult opioid prescriptions in emergency departments (ED) from 2000 to 2010, 3,4 Hoppe et al conducted a large retrospective study on patients with acute pain who were discharged from an urban ED. ${ }^{5}$ The study was undertaken to discern how acute pain management could contribute to recurrent or long-term opioid use. Overall, 17\% of opioid-naïve ED patients who were prescribed and filled their opioid prescription for acute pain were at an increased risk for additional opioid use at 1-year follow-up. ${ }^{6}$ Thus, "the ED may be an important site of intervention" for better managing acute pain without opioid prescriptions. ${ }^{5}$ Beyond the contemporary opioid epidemic, the principles of medicine dictate physicians avoid overtreating patients. Use of nonpharmacological therapies is warranted due to the evidence supporting their effectiveness and safety. ${ }^{1}$

Acupuncture, a form of complementary and alternative treatment originating from traditional Chinese medicine that involves penetrating the skin with thin, solid, metal needles to produce analgesia and other therapeutic effects, may be an effective nonpharmacological approach to managing pain. ${ }^{1,6-9}$ While a 2012 systematic review failed to draw conclusions regarding acupuncture efficacy, ${ }^{10}$ previous studies have suggested the potential benefits of acupuncture utilization in the ED setting for acute pain management. ${ }^{11-13}$ A study conducted by Grissa et al compared acupuncture to intravenous morphine to treat moderate to severe acute pain in ED patients. Overall, these authors found that acupuncture was associated with more effective and faster pain relief with better tolerance. ${ }^{12}$ Additionally, a retrospective pilot study conducted by Reinstein et al investigated clinical outcomes and acceptability of ED acupuncture. ${ }^{11}$ Their findings identified significant reduction in pain and anxiety scores as well as high acceptability among both medical providers and patients. ${ }^{11}$
Few studies have evaluated the use of acupuncture in the community ED setting for pain management. Our quality improvement study aimed to determine if acupuncture improved acute pain following acupuncture treatment and if it could be used as a nonpharmacological option for pain management.

\section{METHODS}

In this quality improvement project, an acupuncture intervention in patients presenting to an ED with acute pain (ie, new pain or acute pain superimposed on chronic) was implemented and data purposefully collected to assess the impact of acupuncture treatment in the ED setting was retrospectively analyzed.

Prior to study initiation, two ED physicians were shadowed to understand patient flow and to determine which patients presenting to the participating ED might most benefit from acupuncture. The ED is located in an urban Midwestern setting and treats an average of 34,000 patients per year. At the time of the study, $66 \%$ of patient visits to the ED were categorized on the Emergency Severity Index (ESI) scale in the 3-5 range. ESI is an ED triage algorithm used to stratify patients from highest severity (1) to lowest severity (5). ${ }^{14}$ Based on ED physician experience, patients designated with an ESI score of 3 , 4 , or 5 often spend 1.5 to 2 hours in the ED waiting for treatment. Given ESI score and ED wait times, patients with ESI of 3-5 were determined to be the most appropriate candidates for this study. In addition to individual treatment rooms, the study ED had a designated semi-private continuum of care area where any patient (regardless of this study) could sit and wait for their care after being triaged. These spaces were determined to be conducive for treating patients with acupuncture, with the continuum of care area being most conducive to treating several patients at one time in a "group" setting.

Findings from this ED assessment were presented to hospital leadership, who guided the overall direction of the project, approved the implementation plan, confirmed the project's goals, and provided resources for project initiation. The project was determined to be a quality improvement study (fulfilling criteria for non-human subjects research) by our health system's institutional review board. Following pilot project 
approvals, the plan for pragmatically implementing acupuncture in the ED setting and for embedding an acupuncturist in the ED included:

1. Providing an in-service presentation to all work team members to garner acceptability. The work team members consisted of ED nurse supervisors, physicians, physician assistants, and support staff.

2. Developing an ED acupuncture competency to ensure the acupuncturists working in this setting would be competent in understanding patient flow in the ED, ESI designations, evidence-based treatment strategies for common conditions presenting to the ED, adhering to ED acupuncture procedures for quality patient-centered care, and collecting pre- and post-acupuncture patient responses for analysis of the efficacy of acupuncture treatments in the ED.

3. Hiring and onboarding a 0.5 full-time equivalent acupuncturist to begin offering acupuncture services in the ED starting January 2017.

4. Analyzing acupuncture data and presenting results to health system leadership to determine acceptability and efficacy of the project in order to provide a foundation for future actions.

Table 1. Workflow and Patient Eligibility for Emergency Department Acupuncture Treatment

1. Upon arrival to the emergency department (ED), all patients triaged by the ED nurse.

2. Patient given Emergency Severity Index $\left(\mathrm{ESI}^{*}\right)$ number $(1-5) ; 1=$ highest severity.

3. Patient's ESI designation and reason for ED visit displayed on ED monitor.

4. Through use of the ED monitor, the ED acupuncturist* determined if the patient would potentially benefit from acupuncture based on the patient's reason for visit and ESI designation, with patients with ESI scores ${ }^{\dagger}$ of 3-5 deemed to be the most likely good candidates for acupuncture.

5. If ED acupuncturist determined patient may benefit from acupuncture, acupuncturist discussed with attending physician or advanced practice provider.

6. If physician or advanced practice provider agreed with ED acupuncturist, ED acupuncturist approached patient to inform about the utilization of acupuncture to help address their chief complaint, including but not limited to pain and/or anxiety.

7. The physician or advanced practice provider let the patient know that utilizing the voluntary acupuncture services came at no cost to them.

8. If patient verbally agreed to ED acupuncture, patient was provided an ED acupuncture clinical "Informed Consent for Treatment" to sign.

9. If patient agreed to treatment and signed the informed consent, patient was transferred to the continuum of care area (CCA) to receive acupuncture in a group acupuncture setting; the CCA consisted of 6 recliner chairs and was regularly staffed by a nurse.

10. Within the CCA, the ED acupuncturist performed an ED intake evaluation for patient's chief complaints and comorbidities; there was a private room adjacent to the CCA that the evaluation could take place in.

11. As part of intake evaluation, pre- and post-acupuncture treatment, patient asked to self-report:

- Level of pain using a 0-10 scoring scale.

- Level of stress using a 0-10 scoring scale.

- Level of anxiety using a 0-10 scoring scale.

- Level of nausea using a 0-10 scoring scale.

12. ED acupuncturist determined acupuncture point of care and documented acupuncture treatment, including number of needles used and placement of needles.

- Needle retention: 20-30 minutes.

13. All patient information and responses to treatments documented in electronic health record.

14. All patients provided information on where to find follow-up acupuncture services.

*Note, the acupuncturist was not on site 24/7; acupuncture services only provided when ED acupuncturist on site.

${ }^{\dagger}$ Acupuncture service was not limited based on ESI; patient's physician had the final say as to recommendation of treatment. 
Additionally, a plan for identifying eligible patients was developed. Table 1 describes patient work flow and eligibility criteria. Ultimately, adult patients were recruited from January 1, 2017, to December 14, 2017, for acupuncture services based on their ESI score, reason for visit, and physician approval. A poster was displayed in the ED and used as an additional recruitment method for alerting patients about the availability of acupuncture in the ED. Prior to and following acupuncture services, patients were asked to self-report their pain, stress, anxiety, and nausea on a scale from none (0) to worst (10).

\section{Statistics}

Characteristics of patients who utilized acupuncture services were obtained retrospectively from the electronic medical record and were described using basic summary statistics. Paired t-tests were used to examine differences between pre- and post-acupuncture pain, stress, anxiety, and nausea scores. Univariable regression analysis was used to identify associations among differences in scores (ie, improved pain, stress, anxiety, and nausea) and patient or visit characteristics including use of opioids or any pain medication during the visit. Significance was associated with a P-value of $\leq 0.05$. All analyses were performed using Minitab ${ }^{\circledR}$ statistical software (Version 13, State College, PA).

\section{RESULTS}

A total of 706 patients were approached in the ED for acupuncture services; 379 (53.7\%) agreed to voluntarily receive acupuncture. Characteristics of patients who utilized acupuncture services are described in Table 2.

Following enrollment, $86.0 \%$ of patients had $8-15$ needles placed and $92.6 \%$ of patients received either 20 or 30 minutes of needle time (mean needle time: 24.4 minutes). Patients mean length of stay in the ED was 210.6 minutes (or $\sim 3.5$ hours). While all patients were discharged from the ED on the same day of their admission, $10.5 \%$ of patients were admitted to the hospital for further care. Only $2.1 \%$ of patients presented to the ED twice within 24 hours, and none received acupuncture services more than once. Following discharge from the ED, less than $2 \%$ of patients received follow-up acupuncture services within 30 days.
Table 2. Characteristics of Patients Who Utilized Acupuncture Services

\begin{tabular}{|c|c|}
\hline \multicolumn{2}{|l|}{ Patient Characteristics $(\mathrm{N}=379)$} \\
\hline Age in years, mean (SD) & $47.5(17.1)$ \\
\hline Female gender, $\mathrm{n}(\%)$ & $258(68.1 \%)$ \\
\hline Body mass index in $\mathrm{kg} / \mathrm{m}^{2}$, mean (SD) & $30.8(8.6)$ \\
\hline \multicolumn{2}{|l|}{ Race/Ethnicity* } \\
\hline White non-Hispanic, n (\%) & $291(77.0 \%)$ \\
\hline Black non-Hispanic, n (\%) & $37(9.8 \%)$ \\
\hline White Hispanic, n (\%) & $45(11.9 \%)$ \\
\hline Asian, n (\%) & $2(0.5 \%)$ \\
\hline American Indian or Alaskan Native, n (\%) & $3(0.8 \%)$ \\
\hline \multicolumn{2}{|l|}{ ED Visit Characteristics ( $N=379)$} \\
\hline \multicolumn{2}{|l|}{ Emergency Severity Index } \\
\hline Score of $2, \mathrm{n}(\%)$ & $22(5.8 \%)$ \\
\hline Score of $3, \mathrm{n}(\%)$ & $261(68.9 \%)$ \\
\hline Score of $4, n(\%)$ & $94(24.8 \%)$ \\
\hline Score of $5, n(\%)$ & $2(0.5 \%)$ \\
\hline \multicolumn{2}{|l|}{ Pain medication utilization during ED visit } \\
\hline Opioids, n (\%) & $176(46.4 \%)$ \\
\hline Nonsteroidal, n (\%) & $104(27.4 \%)$ \\
\hline Tramadol, n (\%) & $6(1.6 \%)$ \\
\hline Any, n (\%) & $238(62.8 \%)$ \\
\hline \multicolumn{2}{|l|}{ Pain medication prescribed following discharge } \\
\hline Opioids, n (\%) & $103(27.2 \%)$ \\
\hline Nonsteroidal, n (\%) & $53(14.0 \%)$ \\
\hline Tramadol, n (\%) & $17(4.5 \%)$ \\
\hline Any, n (\%) & $156(41.2 \%)$ \\
\hline
\end{tabular}

Table 3. Final Pain-Related Diagnosis $(\mathrm{N}=379)$

\begin{tabular}{lc}
\hline Type of Pain & $\mathbf{n}(\%)$ \\
\hline Neck/back pain $^{\mathrm{a}}$ & $133(35.1)$ \\
Abdominal pain $^{\mathrm{b}}$ & $123(32.5)$ \\
Chest pain $^{\mathrm{c}}$ & $35(9.2)$ \\
Head pain $^{\mathrm{d}}$ & $37(9.8)$ \\
Joint/limb pain $^{\text {Substance abuse/withdrawal pain }}$ & $31(8.2)$ \\
Generalized pain $^{\mathrm{C}}$ & $6(1.6)$ \\
\hline
\end{tabular}

anclusive of shoulder pain and hip pain.

${ }^{b}$ Inclusive of urinary tract infection and gastric pain. cInclusive of anxiety- and hypertension-related pain. 'Inclusive of headache, Bell's palsy, epistaxis, and trigeminal neuralgia.

eInclusive of all-over pain or pain in more than one site 
Table 4. Comparison of Mean Scores* Pre- and Post-Acupuncture Treatment

\begin{tabular}{lcccc}
\hline & $\begin{array}{c}\text { Pre-Acupuncture } \\
\text { Treatment }\end{array}$ & $\begin{array}{c}\text { Post-Acupuncture } \\
\text { Treatment }\end{array}$ & \multicolumn{2}{c}{$\begin{array}{c}9 \% \text { CI for Mean } \\
\text { Difference }\end{array}$} \\
\hline Pain score, mean (SD) & $6.5(3.0)$ & $3.4(2.9)$ & $<0.001$ & $3.1(2.8-3.3)$ \\
Stress score, mean (SD) & $5.7(3.9)$ & $1.9(2.6)$ & $<0.001$ & $3.8(3.4-4.1)$ \\
Anxiety score, mean (SD) & $4.8(4.1)$ & $1.6(2.5)$ & $<0.001$ & $3.2(2.9-3.5)$ \\
Nausea score, mean (SD) & $1.6(2.9)$ & $0.6(1.7)$ & $<0.001$ & $1.0(0.78-1.2)$ \\
\hline
\end{tabular}

*Pre- and post-acupuncture scores were available for pain $(N=377)$, stress $(N=378)$, anxiety $(N=375)$, and nausea $(N=373)$. $S D$, standard deviation.

Patients had a final acute pain-related diagnosis primarily for neck/back pain (35.1\%), abdominal pain $(32.4 \%)$, and chest pain $(9.8 \%)$ (Table 3$)$. All post-acupuncture scores were significantly improved compared to pre-acupuncture $(\mathrm{P}<0.001)$, with average pain, stress, anxiety, and nausea scores decreasing by $47.7 \%, 66.6 \%, 66.6 \%$, and $62.5 \%$, respectively (Table 4). In univariable regression analysis, only increasing patient age was significantly associated with improved pain scores $(\mathrm{t}=4.48 ; \mathrm{P}<0.001$; regression equation: improved pain $=1.54+0.0323$ [age]). Patient gender, race/ethnicity, and body mass index had no association with improved pain scores ( $\mathrm{P} \geq 0.50$ for all); therefore, multivariable analysis was not performed. Univariable regression revealed receiving any pain medication during the ED visit was not associated with improved pain scores ( $\mathrm{t}=$ 0.04; $\mathrm{P}=0.693$ ). Receiving only opioids during the $\mathrm{ED}$ visit was not associated with improved pain scores $(\mathrm{t}=-0.06 ; \mathrm{P}=0.948)$.

\section{DISCUSSION}

This study was undertaken to explore the feasibility of providing acupuncture to ED patients with acute pain and to observe how well acupuncture would be received by patients and physicians as an adjunctive or optional nonpharmacological pain management medical intervention. Moreover, this study aimed to determine if acupuncture would reduce self-reported pain, stress, anxiety, and nausea scores.

Our study found that more than half (53.7\%) of ED patients who were encountered and offered acupuncture by the acupuncturist consented to receive treatment. Additionally, their pain, regardless of whether they received any analgesics, was significantly reduced (47.7\% reduction). Likewise, stress, anxiety, and nausea scores were significantly reduced by $>60 \%$. Ultimately, our findings suggest that acupuncture may be an alternative nonpharmacological treatment option for patients who present to the ED.

These results are similar to the pragmatic study of Reinstein et al (set in Minneapolis, Minnesota), who retrospectively analyzed 182 of 217 ED patients treated with acupuncture who did not have missing outcome data. ${ }^{11}$ They found significant reductions from preto post-acupuncture pain and anxiety scores, and no significant difference in pain score changes between patients with and without analgesics administered in the ED. ${ }^{11}$ Reinstein et al also documented a higher participation rate (88.9\% vs our study's $53.7 \%$ ) among patients when they were offered acupuncture by the acupuncturist. ${ }^{11}$ Even so, patients in the previous study may have had a more formal clinician provider referral prior to being approached by the acupuncturist and, thus, more patient/provider acceptance for acupuncture. The participation rate seen in our study was more similar to that of Arnold et al (43.0\%) and Zhang et al (69.0\%), whose studies lacked a formal clinician provider referral. ${ }^{13,15}$

Other studies also have identified significant reductions in pain. Grissa and colleagues randomized 300 eligible adult patients with acute pain presenting to an ED in Tunisia to receive either open-label morphine or acupuncture treatment. ${ }^{12}$ Success in reducing pain scores by $50 \%$ or more was significantly greater in the acupuncture group $(92 \%)$ than in the morphine group $(78 \%)$, and 
resolution time was faster in the acupuncture group (16 minutes vs 28 minutes, respectively). ${ }^{12}$ Another study conducted by Zhang and colleagues that examined 200 Australian ED patients with pain and/ or nausea concluded that acupuncture was feasible and significantly reduced pain and nausea scores. ${ }^{13}$ In a somewhat related Chinese study, in settings where patients go directly to a hospital when ill, acupuncture was found to be effective in relieving acute migraine pain when compared to two different sham acupuncture control groups. ${ }^{9}$

\section{Limitations}

In that this was not a randomized sham-controlled trial, there are many inherent limitations to the quality improvement analysis presented here. This project was only implemented within one urban ED and in patients who had mild to moderate pain and were consented to receive acupuncture treatments. It is not generalizable. Additionally, patients' self-reported scores were based on each individual's perceived and tolerable level of pain, stress, anxiety, and nausea. Furthermore, participants may have reported improved scores given that they were more receptive to acupuncture treatment, increasing the likelihood of selection bias. Even with such limitations, this study had several strengths, including pragmatic design, moderately large sample size, and completed data for $99 \%$ or more of all outcomes.

\section{CONCLUSIONS}

Our study results suggest that patients admitted to the emergency department are accepting of acupuncture and, when provided acupuncture services for acute pain, experience significant decreases in pain, stress, anxiety, and nausea. These quality improvement findings provide a foundation for pursuing more rigorous studies (eg, randomized control trials) in other ED settings to capture a more diverse patient population and determine if providing acupuncture in the ED is an acceptable nonpharmacological treatment option for pain reduction. Nonpharmacological treatment options like acupuncture may decrease prescription narcotics in the ED setting and, arguably, play a role in preventing opioid addiction. Ultimately, while further study is warranted, offering acupuncture in the ED setting appears to improve current pain management among patients with acute pain.

\section{Patient-Friendly Recap}

- Patients being treated in the emergency room may be given opioids to reduce their pain, increasing the risk of additional use after discharge.

- Acupuncture, a form of nonpharmacological treatment, is commonly used to manage patients with chronic pain.

- The authors tested a quality improvement intervention in which acupuncture was offered in the emergency setting to relieve patients' acute pain.

- They found that patient-reported pain, stress, and anxiety scores all significantly improved after acupuncture, with similar benefits seen regardless of whether any pain medication also was received.

\section{Acknowledgments}

The authors thank Michael Urban, MD, Sean Nolan, DO, and Kristen Nitka, RN, for clinical oversight, as well as Jeff Dusek, $\mathrm{PhD}$, and Kevin Greve, MS, for technical assistance.

\section{Author Contributions}

Study design: all authors. Data acquisition or analysis: all authors. Manuscript drafting: all authors. Critical revision: all authors.

\section{Conflicts of Interest}

None.

\section{References}

1. Tick H, Nielsen A, Pelletier KR, et al. Evidence-based nonpharmacologic strategies for comprehensive pain care: the Consortium Pain Task Force white paper. Explore (NY). 2018;14:177-211. CrossRef

2. U.S. Department of Health and Human Services. What is the U.S. opioid epidemic? Updated 2018 Mar 6. https://www.hhs.gov/ opioids/about-the-epidemic/index.html. Accessed July 12, 2018.

3. Mazer-Amirshahi M, Mullins PM, Rasooly I, van den Anker J, Pines JM. Rising opioid prescribing in adult U.S. emergency department visits: 2001-2010. Acad Emerg Med. 2014;21:23643. $\underline{\text { CrossRef }}$

4. Chang HY, Daubresse M, Kruszewski SP, Alexander GC. Prevalence and treatment of pain in EDs in the United States, 2000 to 2010. Am J Emerg Med. 2014;32:421-31. CrossRef

5. Hoppe JA, Kim H, Heard K. Association of emergency department opioid initiation with recurrent opioid use. Ann Emerg Med. 2015;65:493-9. CrossRef

6. National Center for Complementary and Integrative Health. Acupuncture. Last modified 2017 Sep 24. https://nccih.nih. gov/health/acupuncture. Accessed July 12, 2018. 
7. Dhond RP, Yeh C, Park K, Kettner N, Napadow V. Acupuncture modulates resting state connectivity in default and sensorimotor brain networks. Pain. 2008;136:407-18. CrossRef

8. Hasegawa TM, Baptista AS, de Souza MC, Yoshizumi AM, Natour J. Acupuncture for acute non-specific low back pain: a randomised, controlled, double-blind, placebo trial. Acupunct Med. 2014;32:109-15. CrossRef

9. Li Y, Liang F, Yang X, et al. Acupuncture for treating acute attacks of migraine: a randomized controlled trial. Headache. 2009;49:805-16. CrossRef

10. Kim KH, Lee BR, Ryu JH, Choi TY, Yang GY. The role of acupuncture in emergency department settings: a systematic review. Complement Ther Med. 2013;21:65-72. CrossRef

11. Reinstein AS, Erickson LO, Griffin KH, et al. Acceptability, adaptation, and clinical outcomes of acupuncture provided in the emergency department: a retrospective pilot study. Pain Med. 2017;18:169-78. CrossRef

12. Grissa MH, Baccouche H, Boubaker H, et al. Acupuncture vs intravenous morphine in management of acute pain in the ED. Am J Emerg Med. 2016;34:2112-6. CrossRef
13. Arnold AA, Ross BE, Silka PA. Efficacy and feasibility of acupuncture for patients in the ED with acute, nonpenetrating musculoskeletal injury of the extremities. Am J Emerg Med. 2009;27:280-4. CrossRef

14. Agency for Healthcare Research and Quality. Emergency Severity Index (ESI): a triage tool for emergency departments. Agency for Healthcare Research and Quality. Originally posted 2012 Sep; last reviewed 2018 May. https://www.ahrq. gov/professionals/systems/hospital/esi/index.html. Accessed July 12, 2018.

15. Zhang AL, Parker SJ, Smit de V, Taylor DM, Xue CC. Acupuncture and standard emergency department care for pain and/or nausea and its impact on emergency care delivery: a feasibility study. Acupunct Med. 2014;32:250-6. CrossRef

(C) 2019 Aurora Health Care, Inc. 